\title{
A CMOS/Thin-Film Fluorescence Contact Imaging Microsystem for DNA Analysis
}

\author{
Ritu Raj Singh, Student Member, IEEE, Derek Ho, Student Member, IEEE, Alireza Nilchi, Student Member, IEEE,
} Glenn Gulak, Member, IEEE, Patrick Yau, and Roman Genov, Member, IEEE

\begin{abstract}
A hybrid CMOS/thin-film microsystem for fluorescence contact imaging is presented. The microsystem integrates a high-performance optical interference filter and a $128 \times 128$ pixel active pixel sensor fabricated in a standard $0.35-\mu \mathrm{m}$ CMOS technology. The thin-film filter has an optical density greater than 6.0 at the wavelength of interest, providing adequate excitation rejection to the 532-nm solid-state laser. Microsystem performance is experimentally validated by imaging spots of Cyanine-3 fluorophore, conventionally used in DNA detection. The emission intensity as a function of fluorophore concentration is measured with an estimated sensitivity of 5000 fluorophore $/ \mu \mathrm{m}^{2}$. A human DNA microarray has been imaged with the sensor prototype.
\end{abstract}

Index Terms-Contact imaging, DNA detection, fluorescence imaging, microarray, optical filter.

\section{INTRODUCTION}

$\mathbf{M}$ EDICAL, environmental, and life science applications demand for low-cost small-form-factor biochemical sensory systems [1]. Increased portability due to miniaturization enables a higher throughput and a larger range of applications in biochemical detection, particularly microarray-based genetic analysis [2]-[4].

Optical techniques such as fluorescence imaging are commonly used in conventional biochemical sensing instruments [5]. In fluorescence-based sensing, a single molecule of interest can emit millions of photons per second, which is detected by a photodetector, making this technique highly sensitive [6]. Fluorescence-based sensing involves molecular probes, often referred to as fluorophores. These fluorophores are chemically bound to a biochemical to act as a label. When excited by light, fluorophores emit secondary light at a longer wavelength.

In applications such as DNA detection, fluorophores are chemically bound to single-stranded unknown DNA (to be identified), which is then hybridized with a planar array of single-stranded known DNA [7]. After the hybridization process, the extent to which the known and unknown DNA strands bond can be quantified by measuring the intensity of the

Manuscript received October 01, 2009; revised December 23, 2009; accepted January 19, 2010. First published March 29, 2010; current version published May 21, 2010. This work was supported by the Ontario Center of Excellence (OCE) and the National Sciences and Engineering Research Council of Canada (NSERC). This paper was recommended by Associate Editor Y. Massoud.

R. R. Singh, D. Ho, A. Nilchi, G. Gulak, and R. Genov are with the Department of Electrical and Computer Engineering, University of Toronto, Toronto, ON M5S 3G4, Canada (e-mail: roman@eecg.utoronto.ca).

P. Yau is with the University Health Network Microarray Centre, Toronto, ON M5G 1L7, Canada (e-mail: pyau@uhnresearch.ca).

Digital Object Identifier 10.1109/TCSI.2010.2043990



(a)

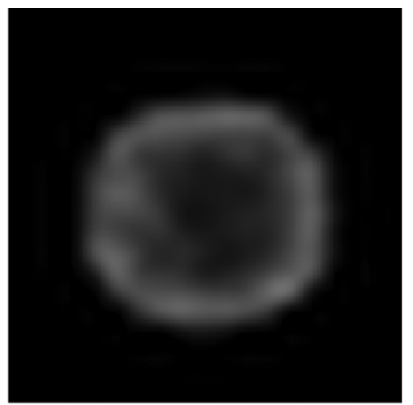

(b)
Fig. 1. Eight-thousand-spot human DNA microarray images. (a) Four clusters of DNA spots. (b) An individual spot on the microarray.

secondary light emitted from the fluorophores. Cyanine-3 (Cy3) and Cyanine-5 (Cy5), which are widely adopted fluorophores for DNA analysis, are conventionally used as fluorescent labels. Fig. 1 shows images of a human DNA microarray containing a total of 8000 DNA spots captured by a commercial scanner from Agilent. Fig. 1(a) depicts four clusters of DNA spots on the microarray. Fig. 1(b) depicts an individual spot on the microarray. After the image is obtained by the microarray scanner, software is utilized to identify the spots in the microarray image and quantify the intensity of each spot.

In a conventional DNA imaging system, the signal path from the excitation source to the detector often involves bulky and expensive optical components such as a system of lenses. Contact imaging, in contrast, is a compact and low-cost technique that involves placing the object to be imaged in close proximity to the photodetector array [8]. It does not require intermediary optics, resulting in significant size reduction, cost savings, and orders of magnitude improvement in sensitivity [9]. These advantages make the contact imaging microsystem attractive for portable low-cost biosensor applications.

Fig. 2 shows a simplified cross section of a fluorescence contact imaging system. An optical filter (A), known as an emission filter, is placed on top of the photodetector die. The fluorophore-labeled analyte is spotted on top of the optical filter. A laser light (B) excites the analyte spots containing the fluorophores. The excited fluorophores emit a secondary light (C) with a longer wavelength. The optical filter blocks the shorter wavelength excitation light of the laser while allowing the longer wavelength emission light to pass through and be sensed by the photodetector.

Emission filter design is often challenging as conventional fluorescence dyes such as $\mathrm{Cy} 3$ have a small difference in the wavelength between the peak excitation and emission spectra, 


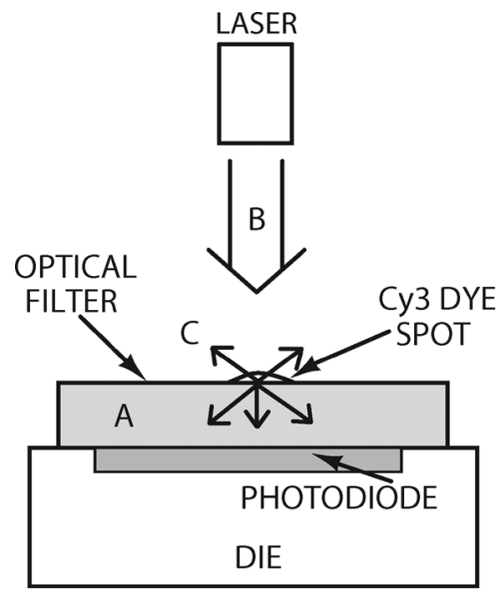

Fig. 2. Fluorescence contact imaging configuration.

known as the Stokes shift. Imaging conventional fluorescence dyes requires an optical filter with a steep cutoff to sufficiently block and transmit the excitation and emission light, respectively. The attenuation property of an optical filter for an out-ofband wavelength is characterized by its optical density (OD) at that wavelength. OD is defined as the ratio of the intensity of light incident on the filter to the intensity of the light that passed through it, in the common logarithmic scale. Specialized fluorophores such as quantum dots have a larger Stokes shift, which relaxes the requirements on the steepness of the filter cutoff [10], but are not typically utilized in many applications such as DNA detection.

To provide high OD, multilayer dielectric interference filters can be used. The optical rejection of such filters is, however, sensitive to the angle of incidence of the excitation light. To prevent filter performance degradation, a collimating lens or a collimated excitation source such as a laser can be utilized.

The choices of the photodetector for fluorescence imaging systems have conventionally been the photomultiplier tube (PMT) and the charge-coupled device (CCD). PMTs are among the most sensitive photodetectors but are bulky and expensive and require high operation voltage, making them unattractive to be integrated into a miniaturized system. The throughput of PMT-based detection systems is relatively low due to the lack of parallelism in a single-photodetector-based PMT. On the other hand, CCDs can be employed in an arrayed implementation but do not allow for on-chip integration of peripheral circuits such as for signal conditioning. This increases the cost and limits miniaturization. Implementations in other semiconductor integration technologies have also been reported, including the use of costly custom silicon-based technologies to implement a single photodetector [11]-[14] and an aluminum gallium arsenide technology to implement a laser as the excitation source [15]. These results are significant but do not yield a low-cost arrayed imager with integrated signal processing.

CMOS technology, on the other hand, has the advantages of low cost, high integration density, and signal processing versatility. It can be efficiently utilized to implement contact imaging arrays with on-chip signal conditioning and processing capabilities. Recently, several proof-of-concept fluorescence contact imaging systems employing CMOS technology and high-performance emission light filters have been reported. An off-theshelf emission filter with a nine-pixel photodetector was reported in [16]. A CMOS web camera was integrated with an optical filter in [17].

Several research groups have reported integration of CMOS photosensors with low-cost emission filters with suboptimal excitation rejection performance. The applications include brain neural activity monitoring [18], particle and pathogen detection [19], [20], and DNA detection [10]. The filters in these designs are generally fabricated directly on the surface of a CMOS die. In such on-CMOS fabrication methods, due to process variation and the fact that the CMOS technology is in general not well characterized for filter deposition, filter performance is often difficult to ensure and cannot readily be verified prior to system integration. This results in an insufficient excitation blocking and reduced sensitivity. The suboptimal filter performance is inadequate for clinical applications such as DNA detection that require stringent excitation rejection with high OD. This can be remedied by filterless fluorescence sensing techniques such as time-resolved fluorescence detection [10] but at the cost of a reduced signal, a larger pixel, and increased design complexity.

Fluorescence imaging microsystems with applications similar to those of the proposed microsystem have recently been reported. The time-resolved Forster-resonance-energy-transfer (TR-FRET) assay reported in [21] performs fluorescence imaging with quantum dots, a relatively new class of fluorescent label, which relax the high-filter-OD requirement. Quantum dots, however, are not widely adopted for DNA analysis. The cellular metabolism fluorometer reported in [22] employs a benzatriazole-PDMS filter [23] with an OD around 3.5. The optical rejection of the filter is sufficient when employing a weak excitation source such as an LED, which compromises emission light detection sensitivity. Although the filter used in [24] has good performance, the high transistor count of this design yields a large pixel size, which limits spatial resolution of DNA spot imaging and on-chip signal processing capabilities.

We present a high-OD low-cost contact imaging microsystem for accurate detection of fluorophores with Stokes shift as small as a few tens of nanometers, such as the conventional $\mathrm{Cy} 3$ dye. It consists of a thin-film interference filter that is prefabricated utilizing proven fabrication methods, with its optical performance verified prior to integration with a CMOS die. A human DNA microarray and the $\mathrm{Cy} 3$ fluorescent dye are utilized to validate the performance of the microsystem. Preliminary results have been reported in [25]. On-chip temporal and spatial signal processing is employed to perform excitation light artifact removal and DNA spot contour identification to further improve DNA image quality and analysis accuracy. The microsystem modeling, the fluorescence imaging microsystem components, the fabrication steps involved in microsystem integration, and the experimental results are presented in subsequent sections of this paper.

\section{Theoretical Analysis-Microsystem Modeling}

This section describes the theoretical modeling of the fluorescence imaging microsystem shown in Fig. 2. An estimate of 
the intensity of the emitted light from the fluorescence dye spots can be derived from Beer-Lambert's law given by

$$
T=\frac{I}{I_{0}}=10^{-\epsilon l M}
$$

where $T$ is the transmissivity of light traveling through a medium. It is defined as the ratio of the intensities of incident excitation light $I_{0}$ to the transmitted excitation light $I . \epsilon$ denotes the absorption coefficient of the medium, $l$ is the distance traveled through the medium, and $M$ is its molar concentration in moles per unit volume. In the proposed setup, where planar fluorophore spots are deposited on the surface of a glass slide, the medium length $l$ is negligible.

The fraction of the excitation light intensity absorbed by the planar spot can be expressed as

$$
A=1-T=1-10^{-\epsilon S}
$$

where $S$ is the concentration of fluorophores on the planar spot in moles per unit area.

The fluorescent dye emits photons at a longer wavelength with an efficiency of $\eta_{\text {dye }}$. The amount of photons $N_{\text {ph }}$ falling on the photodetector can be estimated by

$$
N_{\mathrm{ph}}=\eta_{c p} \eta_{\text {filter }\left(\lambda_{e m}\right)} \eta_{\mathrm{dye}} A \frac{I_{0}}{E_{\mathrm{ph}}}
$$

where $\eta_{\text {filter }\left(\lambda_{\text {em }}\right)}$ is the filter transmission at the fluorescence emission wavelength, $\eta_{c p}$ is the collection efficiency of the noncollimated light from the fluorescing spot [26], and $E_{\mathrm{ph}}$ is the photon energy of the excitation, given by

$$
E_{\mathrm{ph}}=\frac{h c}{\lambda_{e x}}
$$

where $h$ is Planck's constant, $c$ is the speed of light, and $\lambda_{\text {ex }}$ is the excitation wavelength. Equation (3) assumes that the amount of excitation reaching the photodetector is negligible compared to the emission. The filter requirement for this assumption to be valid is

$$
\frac{\eta_{\text {filter }\left(\lambda_{\text {ex }}\right)}}{\eta_{\text {filter }\left(\lambda_{\text {em }}\right)}} \ll 1 .
$$

The photodetector current can be expressed as

$$
I_{\mathrm{PD}}=\eta_{\mathrm{pd}} \eta_{f} N_{\mathrm{ph}}
$$

where $\eta_{p d}$ is the photocurrent collection efficiency of the photodetector, and $\eta_{f}$ is the fill factor of the photopixel. The voltage developed on the photodetector at the end of the integration time can be expressed as

$$
V_{\mathrm{PD}}=\frac{I_{\mathrm{PD}} T_{\mathrm{int}}}{C_{\mathrm{PD}}}
$$

where $T_{\mathrm{int}}$ is the integration time of the photodiode, and $C_{\mathrm{PD}}$ is the photodiode capacitance.

Equation (7) is utilized in the presented design to estimate the fluorescence signal from the Cy3 fluorophore spots.

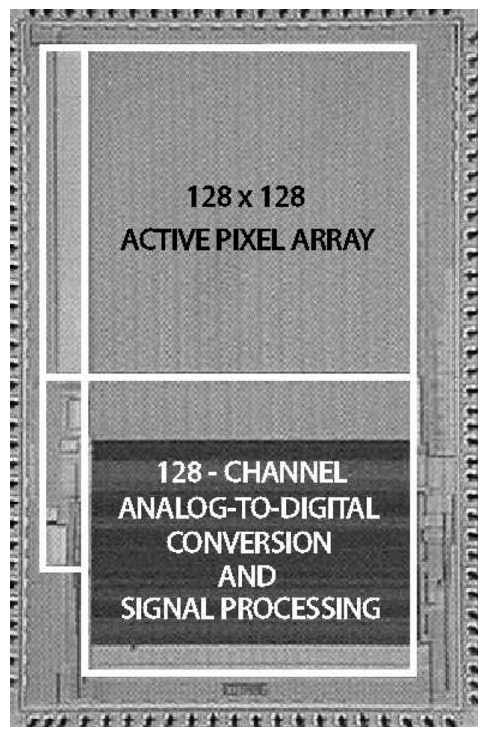

Fig. 3. Chip micrograph of the CMOS contact imager with on-focal-plane signal processing capabilities.

\section{CMOS IMAGER}

A CMOS active pixel sensor was designed for use as the photodetector of the fluorescence imaging microsystem shown in Fig. 2. The imager fabricated in $0.35-\mu \mathrm{m}$ standard CMOS technology can perform on-focal-plane image processing operations such as video compression [27]. Fig. 3 shows the imager die with its $128 \times 128$ photodiode pixel array. The use of CMOS technology significantly reduces the fabrication cost of the photodetector die and also the microsystem integration cost. The circuit implementation of the photodiode pixel array is discussed next.

An array of $128 \times 128$ active pixel sensors transduces the optical signal to an electrical voltage. The pixel and its columnparallel biasing circuit are depicted in Fig. 4. The pixel comprises an $\mathrm{n}^{+}$-diffusion-p-substrate photodiode and two signal paths consisting of two pMOS electronic shutter switches, two frame memories, two column-shared output source followers, and readout switches. At the beginning of each frame, the photodiode is reset by the pMOS reset switch. During the integration period, the $p n$-junction voltage is discharged by an optical current proportional to the incident light intensity. The in-pixel dual frame memory enables the image sensor to perform on-chip temporal and spatial signal processing, as detailed in Section VII.

The pixel area is chosen to be $15.4 \mu \mathrm{m} \times 15.4 \mu \mathrm{m}$ to provide sufficient spatial resolution for imaging the shape of individual microarray spots. The photodiode is implemented as an $\mathrm{n}^{+}$-diffusion-p-substrate structure owing to its compact layout and, hence, a higher pixel fill factor. Reset and shutter transistors are of minimum size as needed to lower channel charge injection and clock feedthrough errors. Using pMOS reset and shutter switches increases the dynamic range of the pixel output. Channel lengths of source follower transistors are larger than the minimum size for good matching among pixels and to reduce the source follower flicker noise. The in-pixel dual frame 


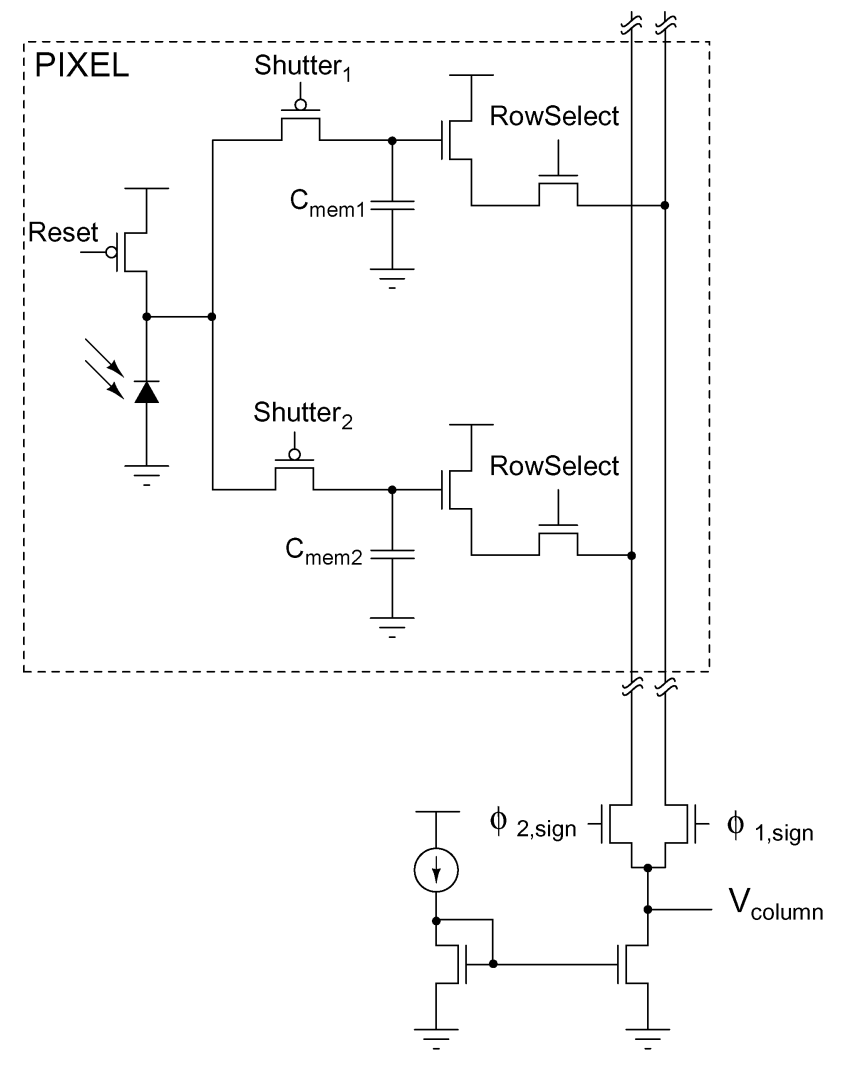

Fig. 4. Pixel circuit with its column-level biasing.



Fig. 5. Column-parallel switched-capacitor difference circuit.

memory is implemented as MOS capacitors to achieve a higher integration density. The MOS capacitors were optimally sized to be $15 \mathrm{fF}$ to achieve a small pixel area, lower charge injection, and clock feedthrough errors. A metal light shield covers the entire pixel, except the photodiode area to eliminate any undesired photo response and to reduce the optical crosstalk from the contact imaging of luminous objects.

Correlated double sampling (CDS) can be performed by the circuit shown in Fig. 5. CDS supresses fixed-pattern noise and flicker noise. This circuit also computes the frame difference for temporal signal processing described in Section VII-A. The amplifier used is a single-stage cascoded common-source nMOS amplifier with a simulated dc gain of $77 \mathrm{~dB}$. Advanced clocking is used to make the charge injection errors signal independent and only appear as a constant offset at the output of the amplifier.

The proposed image sensor also performs on-chip spatial signal processing. A segment of an image is correlated with programmable digital kernels of up to $8 \times 8$ pixels to extract
TABLE I

CMOS IMAGER CHARACTERISTICS

\begin{tabular}{|l|l|}
\hline Technology & $0.35 \mu \mathrm{m}$ CMOS \\
\hline Supply Voltage & $3.3 \mathrm{~V}$ \\
\hline Die Area & $4.4 \mathrm{~mm} \times 2.9 \mathrm{~mm}$ \\
\hline Array Dimensions & $128 \times 128$ pixels \\
\hline Pixel Size & $15.4 \mu \mathrm{m} \times 15.4 \mu \mathrm{m}$ \\
\hline Fill Factor & $28 \%$ \\
\hline Dark Current & $36 \mathrm{fA} /$ pixel \\
\hline Frame Rate & $30 \mathrm{fps}$ \\
\hline Output Resolution & 8 -bit \\
\hline Total Power & $26.2 \mathrm{~mW}$ \\
\hline
\end{tabular}

features of an image, such as edges. This is utilized for DNA spot contour identification in order to improve the quality of DNA analysis, as detailed in Section VII-B. The correlation operation requires computations such as sign transformation, binary-analog multiplication, and accumulation. They are performed by column-parallel multiplying algorithmic analog-to-digital converters, as described in [27].

Table I summarizes the experimentally measured electrical characteristics of the image sensor chip [27].

\section{OPTICAL FILTER}

The optical filter chosen for the microsystem is a discrete thin-film interference filter, which was designed, fabricated (Omega Optical), and optically tested prior to integration with the CMOS die. This approach was chosen compared to a direct deposition of thin-film layers over the CMOS die to ensure the use of proven methods of coating planar substrates during filter fabrication. Direct thin-film deposition over the CMOS die involves higher costs due to complications in adjusting the fabrication process to compensate for the temperature and material differences between the surface of the optical filter and the CMOS die. The masking of bonding pads during the coating process in the direct deposition method adds an additional cost.

In the presented approach, a 100- $\mu$ m-thick 1 in $\times 1$ in optical filter is diced into several smaller pieces of size $2.2 \mathrm{~mm} \times$ $2.8 \mathrm{~mm}$ each (Corwil). The small-sized filter is eventually attached to the CMOS die. The usage of a single original filter during fabrication significantly reduces the cost involved, per piece. The interference filter was fabricated using 60 layers of $\mathrm{Nb}_{2} \mathrm{O}_{5}$ and $\mathrm{SiO}_{2}$. These materials were selected for their durability and for their optical properties. The coatings were deposited onto a $100-\mu \mathrm{m}$-thick microsheet of fused silica substrate by physical vapor deposition. The coated substrate is then cut with a diamond saw to the size required to cover the photosensor array. Finally, the diced filter was laminated to the CMOS die using a refractive-index-matching epoxy.

The interference filter is a thin long-pass optical filter with a cutoff wavelength of $565 \pm 5 \mathrm{~nm}$. The cutoff wavelength was chosen to transmit the emitted light from $\mathrm{Cy} 3$ fluorescent markers. Cy3 fluorphores have an emission light peak at $575 \mathrm{~nm}$. The transmission of the filter is greater than $75 \%$ at $575 \mathrm{~nm}$ and, on the average, greater than $85 \%$ from 575 to $700 \mathrm{~nm}$. The filter 

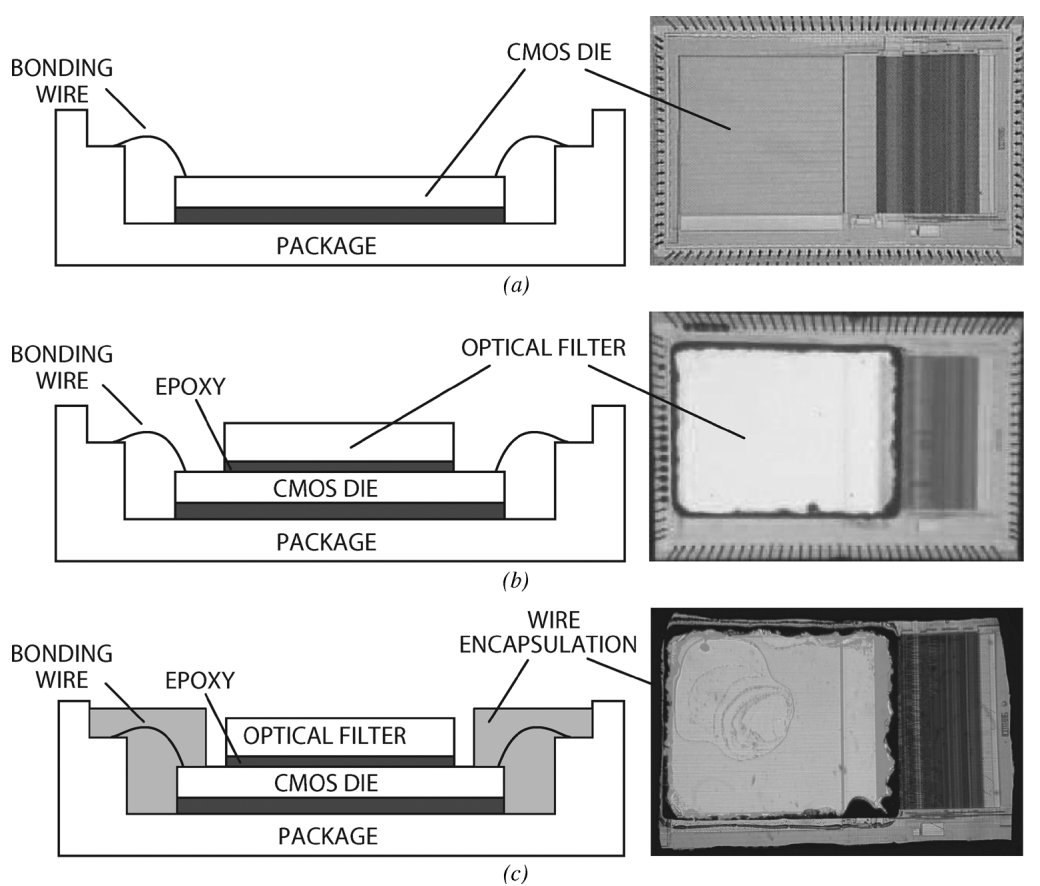

Fig. 6. (Left) Cross-sectional diagram and (right) die micrograph for three key microsystem integration steps. (a) CMOS die is packaged and wire bonded. (b) Diced optical filter is attached to the CMOS die using a refractive-index-matched epoxy. (c) Bonding wires are encapsulated with an epoxy for protection.

is designed to have an OD of 7 at $532 \mathrm{~nm}$, the laser wavelength, and has experimentally been measured to achieve an OD of 6 for an angle of incidence less than $20^{\circ}$. Due to the limitations of the testing equipment, an OD beyond 6 could not be measured.

\section{SYSTEM ASSEMBLY}

Fig. 6 describes the steps in microsystem integration. Fig. 6(a) shows the CMOS die bonded and packaged using a standard wire bonding and packaging process. The CMOS die was raised inside the chip package by placing a die spacer below it. As required for contact imaging, this brings the photosensor closer to the top surface of the chip package, where the microarray slide is placed. Fig. 6(b) shows the attachment of the optical filter to the CMOS die using a refractive-index-matched epoxy. Using an index-matched epoxy eliminates any unwanted light reflections at the filter-CMOS die surface. Finally, in Fig. 6(c), an opaque epoxy dam is built around the optical filter, and epoxy poured over the bonding wires. This bonding wire encapsulation using epoxy electrically insulates the bonding wires, protects them, and restricts stray reflected laser light from leaking through the sides of the filter to reach the sensor array.

\section{EXPERIMENTAL RESULTS}

The fluorescence contact imaging microsystem has experimentally been validated by imaging spots of $\mathrm{Cy} 3$ fluorescent dye (GE Healthcare). Fig. 7 shows the test setup. The optical filter is attached to the CMOS die inside the cavity of an open-lid ceramic chip package mounted on a printed circuit board. The distance from the CMOS die surface to the chip package surface is approximately $1 \mathrm{~mm}$. A custom glass slide with Cy 3 dye spots is placed on the surface of the chip package. A mechanical stage aligns the dye spots over the CMOS pixel array. A 532-nm pen-sized laser with $10-\mathrm{mW}$ light intensity is mounted

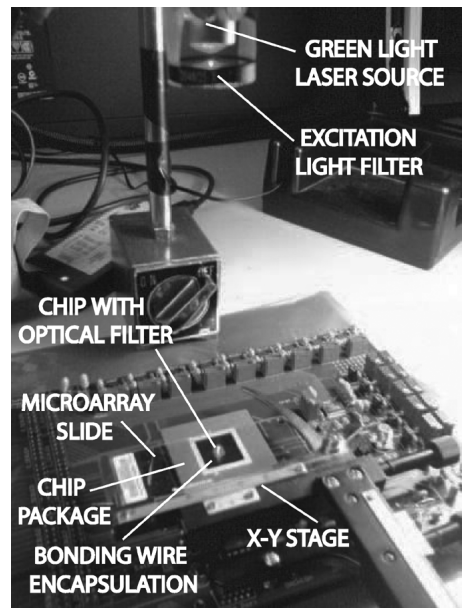

Fig. 7. Fluorescence contact imaging test setup.

vertically above the CMOS pixel array to excite the fluorescent dye spots aligned over the array. The secondary light emitted by the spot is sensed by the imager, while the laser light gets attenuated by the on-chip filter. The laser emits a parasitic $800-\mathrm{nm}$ wavelength that is blocked by an excitation light filter.

Fig. 8 depicts the experimentally measured optical characteristics of the filter, the laser, and the Cy3 fluorescent dye. The OD of the $100-\mu \mathrm{m}$-thick filter (A) was measured prior to its dicing. The laser excitation beam (B) shown onto the Cy3 fluorophores falls within the dye absorption spectrum (D) of the $\mathrm{Cy} 3$ dye. The laser excitation light is attenuated by more than $60 \mathrm{~dB}(\mathrm{OD}>$ 6), while the $\mathrm{Cy} 3$ dye emission (C) reaches the pixel array almost unattenuated at its peak wavelength.

Fig. 9 shows an experimentally captured image of four spots of $10-\mu \mathrm{M}$ concentration Cy3 fluorescent dye. They are spotted 


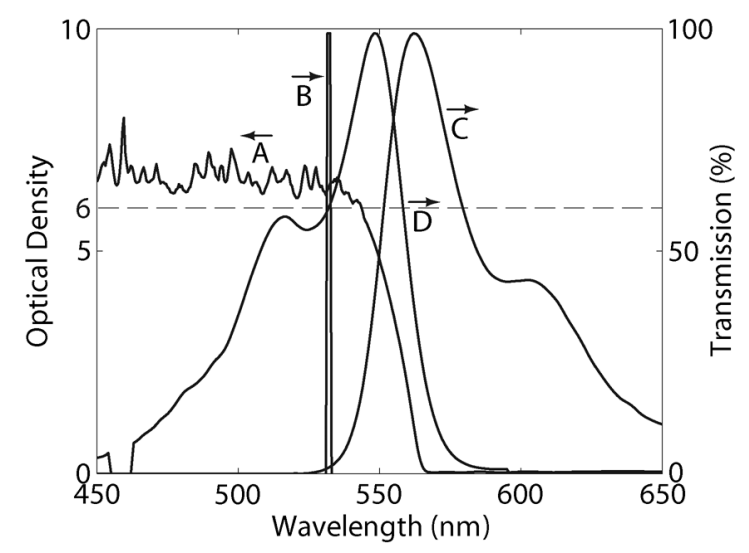

Fig. 8. Measured spectral characteristics: (A) filter optical density, (B) laser excitation, (C) $\mathrm{Cy} 3$ fluorescence emission, and (D) $\mathrm{Cy} 3$ absorption.

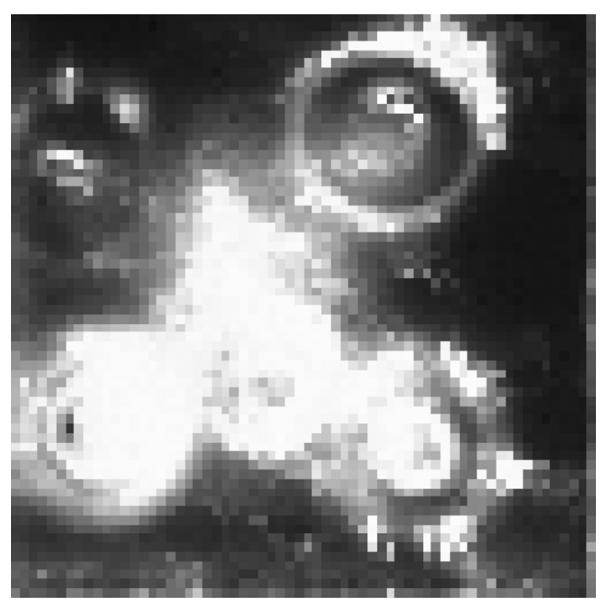

Fig. 9. Experimentally captured image of four spots of $10-\mu \mathrm{M}$ concentration Cy3 fluorescent dye placed $200 \mu \mathrm{m}$ above the CMOS imager surface.

onto a $100-\mu \mathrm{m}$-thin glass slide and placed over the optical filter into the packaging cavity. The spots' fluoresce and their emission are captured by the photosensor. The high-intensity region in the center is likely due to air bubbles between the optical filter and the CMOS die. The air bubbles may be a result of a lack of degassing. Gas is released from the epoxy when it is heated to bond the filter and the sensor. A mismatch in the refractive index at the bottom side of the filter can result in degraded performance of the filter. A solution to this problem is to employ appropriate degassing techniques in order to prevent gas from being trapped during the bonding process. Some additional minor excitation light scattering may be due to a small air gap between the slide and the filter and light scattering within the spot. The scattered light, due to a lack of collimation, cannot effectively be filtered and shows up in the image. Much of the laser light artifact can be removed by on-chip frame differencing, as discussed in Section VII-A.

Fig. 10 shows a bright spot from a 8000-spot human DNA microarray slide imaged by the proposed microsystem. The spot size is $250 \mu \mathrm{m}$. A bright halo ring associated with the fluorescing spot was observed, due to the fact that the microarray slide was placed further away, i.e., $1 \mathrm{~mm}$ above the image sensor surface.

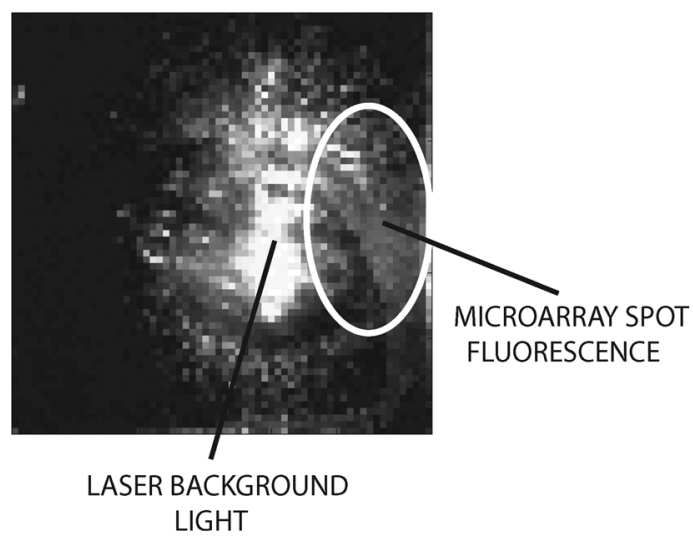

Fig. 10. One spot of an 8000-spot human DNA microarray captured by the presented microsystem at 1-mm distance above the CMOS imager surface.

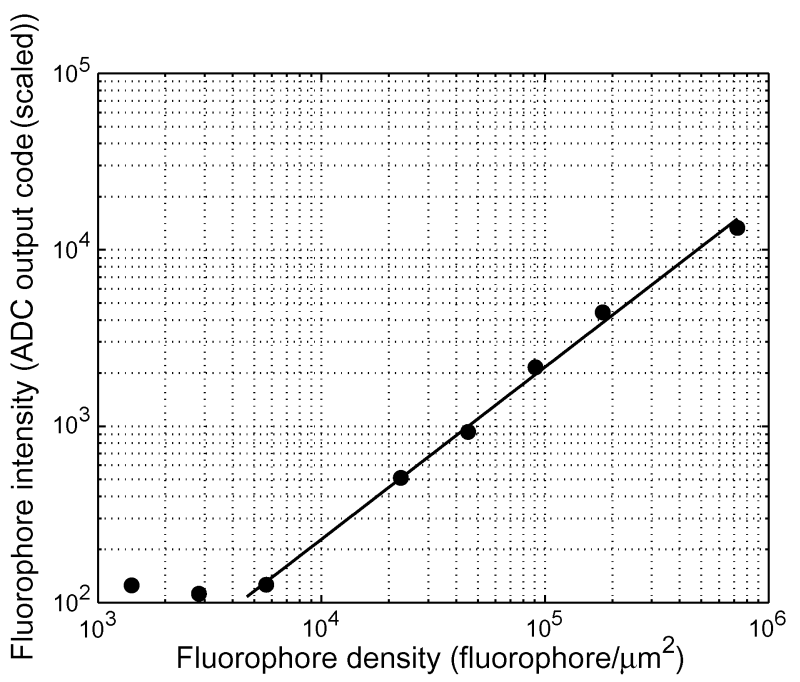

Fig. 11. Experimentally measured light intensity versus the Cy3 fluorophore density.

This distance was constrained by the die packaging configuration. The high-intensity area observed in the middle is likely due to air bubbles trapped in the epoxy. As stated above, epoxy degassing and on-chip frame differencing are available solutions to eliminate this artifact.

Fig. 11 shows the experimentally measured fluorescent light intensity for different concentrations of the $\mathrm{Cy} 3$ fluorescence dye. Volumes of the dilution liquid were measured and mixed with the $\mathrm{Cy} 3$ dye to create binary-weighted fluorophore concentrations from $10 \mu \mathrm{M}$ down to $0.02 \mu \mathrm{M}$. Solutes of different concentrations of the fluorescence dye were spotted on a custom glass slide. The diameter of the dye spots was $2 \mathrm{~mm}$ in order to match the pixel array dimensions. The spot pitch was $2 \mathrm{~mm}$ to avoid crosstalk between the adjacent dye spots. The Cy3 fluorescence dye was preprocessed to stabilize it and lengthen its lifetime (Full Moon Biosystems).

A linear fit has been performed on the measured signal for all fluorophore concentrations. The preparation accuracy of the smaller concentrations was not well controlled due to the large difference in the quantities of the dye and the dilution liquid. Based on the graph, fluorophore concentrations as small as 5000 fluorophores $/ \mu \mathrm{m}^{2}$ can be measured. 


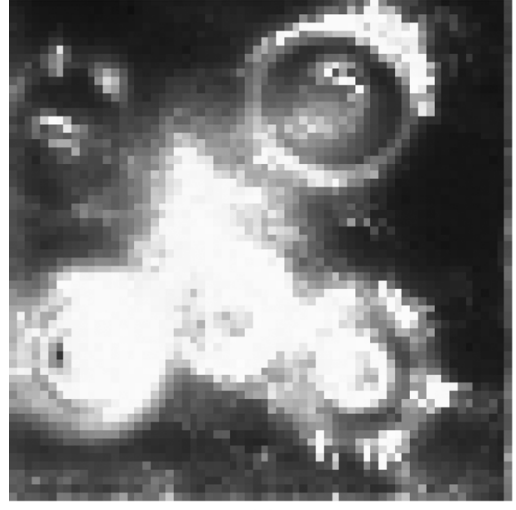

(a)

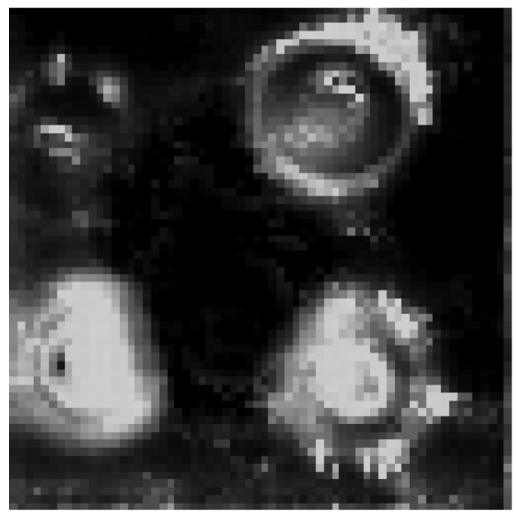

(c)

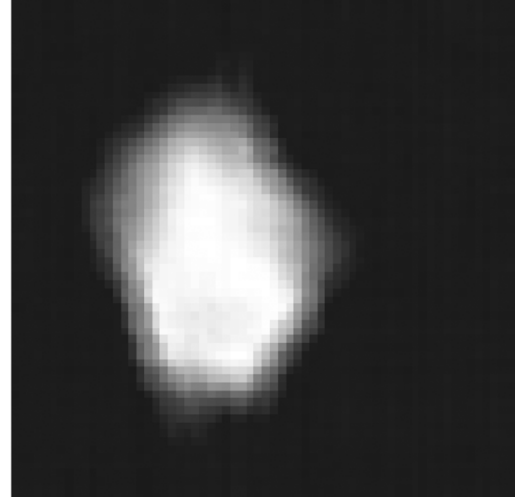

(b)



(d)

Fig. 12. On-chip imaging and identification of Cy3 dye spots. (a) Experimentally obtained image of four Cy3 spots (the same as in Fig. 9). (b) Experimentally obtained image of laser excitation light artifact. (c) Simulated on-chip artifact removal by frame differencing performed on the data shown in subfigures (a) and (b). (d) Simulated on-chip DNA spot contour identification performed on the frame-differenced image shown in subfigure (c).

\section{ON-ChIP SIGNAL PROCESSING}

The proposed CMOS image sensor integrates the capability to perform on-chip signal processing to improve DNA detection performance.

\section{A. On-Chip Temporal Signal Processing}

On-chip frame differencing can be utilized to remove the excitation light artifact that is present due to laser light scattering at the media interfaces and at epoxy nonuniformities such as gas bubbles. Fig. 12(a) shows the image of four $\mathrm{Cy} 3$ spots, previously depicted in Fig. 9 and repeated here for clarity. Fig. 12(b) shows an image taken before a microarray slide is introduced. The image is ideally dark but shows the excitation laser resulting from inadequate filter rejection, likely due to the air bubbles in the epoxy between the filter and the CMOS die. As previously mentioned, another possible cause of the artifact is the air gap between the slide and the filter. To remove this artifact, one solution is to take a background image prior to the placement of the spots over the image sensor. Frame differencing is then performed to subtract the laser light captured in the background image from the spots' image. Frame differencing can be performed by the circuits in Figs. 4 and 5, as detailed in [27]. The spots to be imaged are mechanically moved into the field of view after a background image of an area of the slide without any spots has been sampled. The background signal and the full signal are stored in the dual frame memory of the pixel array. The CDS circuit in Fig. 5 then performs the differencing function. Fig. 12(c) is the simulated difference image, which demonstrates significant laser light suppression. The fluorescent labels show up as four distinct spots upon the removal of the laser light artifact.

\section{B. On-Chip Spatial Signal Processing}

On-chip spatial signal processing can be utilized to provide capabilities currently performed by a dedicated computer in a benchtop microarray imaging instrument. Contour detection, for example, can be used to identify the boundary of a DNA microarray spot. Fig. 12(d) shows an image that resulted from an on-chip contour detection simulation performed on the image in Fig. 12(c). To achieve contour detection, the proposed image sensor computes 2-D convolution of images with a programmable digital kernels of up to $8 \times 8$ pixels in parallel directly on the focal plane, as discussed in Section III. Since DNA microarray spots often have irregular shapes as a result of the microarray fabrication process, gaining knowledge of the spot boundary from contour detection and taking only the area covered by the spot into account leads to improved DNA analysis accuracy.

Experimental validation of the on-chip temporal and spatial signal processing functionalities in the context of video compression was presented in [27]. 
TABLE II

CMOS Fluorescence ContaCt IMAGERS' Comparative ANALYsis

\begin{tabular}{|c|c|c|c|c|}
\hline & [21] & {$[22],[23]$} & {$[24]$} & This work \\
\hline Fabrication process & $0.35 \mu \mathrm{m}$ CMOS & $0.5 \mu \mathrm{m}$ CMOS & $0.35 \mu \mathrm{m}$ CMOS & $0.35 \mu \mathrm{m}$ CMOS \\
\hline Filter type & Interference & Polymer & Interference & Interference \\
\hline $\begin{array}{c}\text { Filter Fabrication } \\
\text { Method }\end{array}$ & $\begin{array}{l}\text { Deposited on } \\
\text { CMOS }\end{array}$ & Prefabricated & $\begin{array}{l}\text { Coupled through } \\
\text { fiber optic face } \\
\text { plate }\end{array}$ & Prefabricated \\
\hline $\begin{array}{l}\text { Filter optical density } \\
\text { at wavelength }\end{array}$ & 3.0-4.0@406nm & $3.5 @ 375 n m$ & $4.9 @ 532 \mathrm{~nm}$ & $>6.0 @ 532 \mathrm{~nm}$ \\
\hline Fluorescence dye & $\begin{array}{c}\text { Qdot } 655 \\
\text { CdSe/ZnS 406nm }\end{array}$ & $\begin{array}{l}\text { Fluorescing } \\
\text { NADH }\end{array}$ & $\begin{array}{l}\text { Conventional } \\
\text { Cy3 dye }\end{array}$ & $\begin{array}{l}\text { Conventional } \\
\text { Cy3 dye }\end{array}$ \\
\hline $\begin{array}{l}\text { Fluorescence dye } \\
\text { peak excitation } \\
\text { wavelength }\end{array}$ & $406 \mathrm{~nm}$ & $380 \mathrm{~nm}$ & $532 \mathrm{~nm}$ & $532 \mathrm{~nm}$ \\
\hline $\begin{array}{l}\text { Fluorescence dye } \\
\text { peak emission } \\
\text { wavelength }\end{array}$ & $655 \mathrm{~nm}$ & $460 \mathrm{~nm}$ & $575 \mathrm{~nm}$ & $575 \mathrm{~nm}$ \\
\hline $\begin{array}{c}\text { Fluorescence dye } \\
\text { stokes shift }\end{array}$ & $249 \mathrm{~nm}$ & $80 \mathrm{~nm}$ & $43 \mathrm{~nm}$ & $43 \mathrm{~nm}$ \\
\hline Excitation source & Laser@655nm & $\begin{array}{c}\text { UV LED @ } \\
375 \mathrm{~nm}\end{array}$ & Laser@532nm & Laser@532nm \\
\hline Photodiode area & $15 \mu \mathrm{m}^{2}$ & $36 \mu \mathrm{m} \times 36 \mu \mathrm{m}$ & $50 \mu \mathrm{m} \times 50 \mu \mathrm{m}$ & $5 \mu \mathrm{m} \times 15.4 \mu \mathrm{m}$ \\
\hline Pixel architecture & $\begin{array}{l}\text { Direct } \\
\text { integration, } \\
\text { differential } \\
\text { readout }\end{array}$ & $\begin{array}{l}\text { Direct } \\
\text { integration, } \\
\text { differential } \\
\text { readout }\end{array}$ & CTIA & Direct integration \\
\hline Pixel size & $40 \mu \mathrm{m} \times 40 \mu \mathrm{m}$ & $34 \mu \mathrm{m} \times 34 \mu \mathrm{m}$ & $250 \mu \mathrm{m} \times 250 \mu \mathrm{m}$ & $15.4 \mu \mathrm{m} \times 15.4 \mu \mathrm{m}$ \\
\hline Array size & $64 \times 64$ & 1 & $7 \times 7$ & $128 \times 128$ \\
\hline $\begin{array}{l}\text { On-chip temporal } \\
\text { signal processing (e.g. } \\
\text { artifact removal) }\end{array}$ & No & No & No & Yes \\
\hline $\begin{array}{l}\text { On-chip spatial } \\
\text { signal processing (e.g. } \\
\text { DNA spot contour } \\
\text { identification) }\end{array}$ & No & No & No & Yes \\
\hline
\end{tabular}

\section{DISCUSSION}

Table II provides a comparison of the implemented fluorescence imaging microsystem with other CMOS/thin-film contact imaging microsystems reported in the literature. The TR-FRET assay reported in [21] employs quantum dots, a relatively new type of label, for fluorescence imaging. Quantum dots, owing to their large Stokes shift, relax the rejection requirements on the optical filter. The filter can therefore have a less steep rejection curve at the cutoff wavelength. However, due to a number of reasons such as their large physical size and possible side effects on DNA chemistry, they are not widely used in DNA analysis. In comparison, the implemented microsystem utilizes the conventionally accepted $\mathrm{Cy} 3$ fluorescence dye.

The cellular metabolism fluorometer reported in [22] employs a benzatriazole-PDMS filter [23] with an OD around 3.5. The optical rejection of the filter is sufficient when employing a weak excitation source such as an LED. The weak excitation, however, results in reduced detection sensitivity, as indicated in (3).
The high transistor count of the design in [24] yields a large pixel size. The small pixel size of the proposed design enables imaging of the spatial geometry details of each spot. This level of detail, combined with on-chip signal processing, enables further improvements in image quality and detection accuracy.

\section{CONCLUSION}

A hybrid CMOS/thin-film microsystem for fluorescence contact imaging has been presented. The optical filter has been fabricated and characterized prior to its integration with the CMOS die, yielding greater control over the filter performance. The microsystem performs fluorescence imaging by exciting microarray spots using an inexpensive off-the-shelf laser. The microsystem has experimentally been validated by imaging the 8000-spot human DNA microarray and the Cy3 fluorescence dye, commonly employed in clinical DNA detection applications. On-chip temporal and spatial signal processing capabilities enable artifact removal and specimen boundary identification, respectively, which further improves DNA analysis quality and yields a compact and low-cost implementation. 


\section{ACKNOWLEDGMENT}

The authors would like to thank Dr. E. Istrate of the Institute of Optical Sciences, Toronto, ON, Canada, D. Upton of Omega Opticals, and N. Stickle of the University Health Network Microarray Centre, Toronto, for their technical support and insightful discussions. The authors also thank Canadian Microelectronics Corporation for providing the fabrication services and microsystem integration services.

\section{REFERENCES}

[1] A. G. Andreou, J. Chen, P. Chung, and S. Wong, "Enabling technologies in drug delivery and clinical care," in Proc. IEEE Int. Symp. Circuits Syst., May 2007, pp. 2874-2877.

[2] I. Lestas, J. Paulsson, N. Ross, and G. Vinnicombe, "Noise in gene regulatory networks," IEEE Trans. Autom. Control, vol. 53, no. 1, pp. 189-200, Jan. 2008.

[3] E. Anderson, J. Daniels, N. Pourmand, and T. Lee, "Crosstalk in integrated microarrays with current sensing," IEEE Trans. Circuits Syst. I, Reg. Papers, vol. 55, no. 11, pp. 3756-3762, Dec. 2008.

[4] Y. Li, K. Kirstein, J. Lichtenberg, and A. Hierlemann, "Monolithic resonant-cantilever-based CMOS microsystem for biochemical sensing," IEEE Trans. Circuits Syst. I, Reg. Papers, vol. 55, no. 9, pp. 2551-2560, Oct. 2008

[5] O. S. Wolfbeis, Fluorescence Methods and Applications, 1st ed. Oxford, U.K.: Blackwell, 2008.

[6] M. Barnes, W. Whitten, and J. M. Ramsay, "Detecting single molecules in liquids," Anal. Chem., vol. 67, pp. 418A-423A, Jul. 1995

[7] R. L. Stears, T. Martinsky, and M. Schena, "Trends in microarray analysis," Nat. Med., vol. 9, no. 1, pp. 140-145, Jan. 2003.

[8] U. C. Fischer and H. P. Zingsheim, "Submicroscopic contact imaging with visible light by energy transfer," Appl. Phys. Lett., vol. 40, no. 3, pp. 195-197, Feb. 1982.

[9] K. Salama, H. Eltoukhy, A. Hassibi, and A. E. Gamal, "Modeling and simulation of luminescence detection platforms," Biosens. Bioelectron., vol. 19, no. 11, pp. 1377-1386, Jun. 2004.

[10] G. Patounakis, K. L. Shepard, and R. Levicky, "Active CMOS array sensor for time-resolved fluorescence detection," IEEE J. Solid-State Circuits, vol. 41, no. 11, pp. 2521-2530, Nov. 2006.

[11] O. Hofmann, X. Wang, A. Cornwell, S. Beecher, A. Raja, D. D. C. Bradley, A. J. deMelloc, and J. deMello, "Monolithically integrated dye-doped PDMS long-pass filters for disposable on-chip fluorescence detection," Lab Chip, vol. 6, no. 8, pp. 981-987, Aug. 2006.

[12] J. Webster, M. Burns, D. Burke, and C. Mastrangelo, "Monolithic capillary electrophoresis device with integrated fluorescence detector," Anal. Chem., vol. 73, no. 7, pp. 1622-1626, Apr. 2001.

[13] M. Burns, B. N. Johnson, S. N. Brahmasandra, K. Handique, J. R. Webster, M. Krishnan, T. S. Sammarco, P. M. Man, D. Jones, D. Heldsinger, C. H. Mastrangelo, and D. T. Burke, "An integrated nanoliter DNA analysis device," Science, vol. 282, no. 5388, pp. 484-487, Oct. 1998.

[14] V. Namasivayam, R. Lin, B. Johnson, S. Brahmasandra, Z. Razzacki, D. T. Burke, and M. A. Burns, "Advances in on-chip photodetection for applications in miniaturized genetic analysis systems," J. Micromech. Microeng., vol. 14, no. 1, pp. 81-90, Jan. 2004.

[15] E. Thrush, O. Levi, K. Wang, J. Harris, and S. Smith, "Integrated semiconductor fluorescent detection system for biochip and biomedical applications," in Proc. IEEE-EMBS Conf., May 2002, pp. 374-379.

[16] S. Parikh, G. Gulak, and P. Chow, "A CMOS image sensor for DNA microarrays," in Proc. IEEE Custom Integr. Circuits Conf., Sep. 2007, pp. 821-824.

[17] L. Martinelli, H. Choumane, K.-N. Ha, G. Sagarzazu, C. Goutel, and C. Weisbuch, "Sensor-integrated fluorescent microarray for ultrahigh sensitivity direct-imaging bioassays: Role of a high rejection of excitation light," Appl. Phys. Lett., vol. 91, no. 8, pp. 083 901-083 901-3, Aug. 2007.
[18] D. C. Ng, H. Tamura, T. Mizuno, T. Tokuda, M. Nunoshita, Y. Ishikawa, S. Shiosaka, and J. Ohta, "An implantable and fully-integrated complementary metal-oxide semiconductor device for in vivo neural imaging and electrical interfacing with the mouse hippocampus," Sens. Actuators, vol. 48, pp. 176-186, Jul. 2008.

[19] D. Sander, M. Dandin, H. Ji, N. Nelson, and P. Abshire, "Lownoise CMOS fluorescence sensors," in Proc. IEEE Int. Symp. Circuits Syst., May 2007, pp. 2007-2010.

[20] M. Beiderman, T. Tam, A. Fish, G. A. Jullien, and O. Y. Pecht, "A low noise CMOS image sensor with an emission filter for fluorescence applications," in Proc. IEEE Int. Symp. Circuits Syst., May 2008, pp. $1100-1103$

[21] D. Schwartz, P. Gong, and K. L. Shepard, "Time-resolved Forster-resonance-energy-transfer DNA assay on an active CMOS microarray," Biosens. Bioelectron., vol. 24, no. 3, pp. 383-390, Nov. 2008.

[22] N. Nelson, D. Sander, M. Dandin, A. Sarje, S. Prakash, H. Ji, and P. Abshire, "A handheld fluorometer for measuring cellular metabolism," in Proc. IEEE Int. Symp. Circuits Syst., May 2008, pp. 1080-1083.

[23] H. Ji, M. Dandin, E. Smela, and P. Abshire, "Integrated fluorescence sensing for lab-on-a-chip devices," in Proc. IEEE/NLM Life Sci. Syst. Appl. Workshop, Jul. 2006, pp. 1-2.

[24] B. Jang, P. Cao, A. Chevalier, and A. Hassibi, "A CMOS fluorescentbased biosensor microarray," in Proc. IEEE Int. Solid-State Circuits Conf., Feb. 2009, pp. 436-437.

[25] R. Singh, D. Ho, A. Nilchi, R. Genov, and G. Gulak, "A hybrid thinfilm/CMOS fluorescence contact imager," in Proc. IEEE Int. Symp. Circuits Syst., May 2009, pp. 2437-2440.

[26] E. Thrush, O. Levi, L. J. Cook, J. Deich, A. Kurtz, S. J. Smith, W. E. Moerner, and J. S. Harris, Jr., "Monolithically integrated semiconductor fluorescence sensor for microfluidic applications," Sens. Actuators B, Chem., vol. 105, no. 2, pp. 393-399, Mar. 2005.

[27] A. Nilchi, J. Aziz, and R. Genov, "Focal-plane algorithmically-multiplying CMOS computational image sensor," IEEE J. Solid-State Circuits, vol. 44, no. 6, pp. 1829-1839, Jun. 2009.

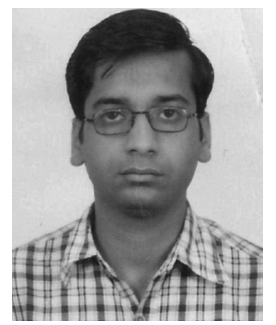

Ritu Raj Singh (S'10) received the B.Tech. degree in electrical engineering from the Indian Institute of Technology Kanpur, Kanpur, India, in 2005, and the M.A.Sc. degree in electrical and computer engineering from the University of Toronto, Toronto, ON, Canada, in 2009.

He has held engineering positions with Granite SemiCom Inc., Toronto, Mentor Graphics, India, and Texas Instruments, India, in 2009, 2005-2006, and 2004, respectively. He is currently with the Department of Electrical and Computer Engineering, University of Toronto. His research interests focus on the development of low-cost miniaturized biosensors utilizing standard CMOS technology.

Mr. Singh received the Proficiency medal for the best undergraduate project in Electrical Engineering in 2005, the National Talent Scholarship in 1999, and the All India Talent Scholarship presented by the Prime Minister of India in 1997.

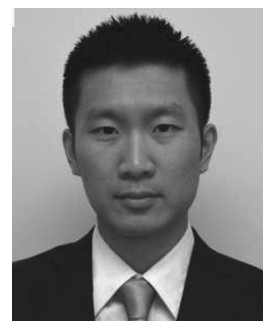

Derek Ho (S'09) received the B.A.Sc. and M.S. degrees from the University of British Columbia, Vancouver, BC, Canada, in 2005 and 2007, respectively. $\mathrm{He}$ is currently working toward the Ph.D. degree on CMOS image sensors for scientific and medical applications in the Department of Electrical and Computer Engineering, University of Toronto, Toronto, ON, Canada.

Mr. Ho was the recipient of the Postgraduate Scholarship and Undergraduate Student Research Award from the Natural Sciences and Engineering Research Council of Canada. 


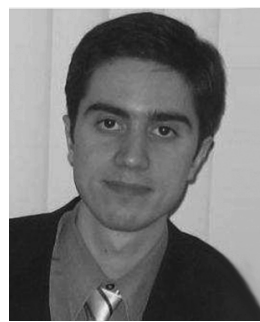

Alireza Nilchi (S'01) received the B.Sc. degree (with honors) in electrical and electronics engineering from the University of Tehran, Tehran, Iran, in 2005 and the M.A.Sc. degree in electrical engineering from the University of Toronto, Toronto, ON, Canada, in 2008, where he is currently working toward the Ph.D. degree in the Department of Electrical and Computer Engineering.

He has held a summer position with the Universität der Bundeswehr München, Munich, Germany, in 2004. His current research interests are on scaled CMOS analog/mixed-signal integrated circuits and very low power data converters for sensor applications.

Mr. Nilchi was the recipient of the Best Student Paper Award of the IEEE Circuits and Systems Conference (ISCAS), as well as a corecipient of the Best Paper Award of the Sensory Systems Technical Committee of the IEEE Circuits and Systems Society in 2009.

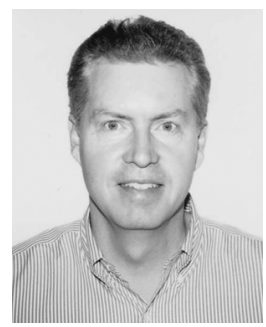

Glenn Gulak (M'90) received the Ph.D. degree from the University of Manitoba, Winnipeg, MB, Canada, while holding a Natural Sciences and Engineering Research Council of Canada Postgraduate Scholarship.

From January 1985 to January 1988, he was a Research Associate in the Information Systems Laboratory and the Computer Systems Laboratory, Stanford University, Stanford, CA. From March 2001 to March 2003, he was the Chief Technical Officer and Senior VP LSI Engineering of a fabless semiconductor startup headquartered in Irvine, CA. He is currently a Professor with the Department of Electrical and Computer Engineering, University of Toronto, Toronto, ON, Canada. He held the L. Lau Chair in Electrical and Computer Engineering for the 5-year term from 1999 to 2004. He currently holds the Canada Research Chair in Signal Processing Systems and the Edward S. Rogers Sr. Chair in Electrical Engineering. He is the author or a coauthor of more than 100 publications in refereed journal and refereed conference proceedings. His current research projects are focused on high-performance MIMO OFDM implementations and in CMOS biosensors. His present research interests are in the areas of algorithms, circuits, and CMOS system-on-chip implementations for digital communication systems and, additionally, in the area of CMOS biosensors.

Dr. Gulak is a registered Professional Engineer in the Province of Ontario. He is the recipient of numerous teaching awards for undergraduate courses taught in both the Department of Computer Science and the Department of Electrical and Computer Engineering at the University of Toronto. He received the IEEE Millennium Medal in 2001. He has served on the International Solid-State Circuits Conference (ISSCC) Signal Processing Technical Subcommittee from 1990 to 1999. He has served as the ISSCC Technical Vice Chair in 2000 and served as the Technical Program Chair for ISSCC 2001. He served on the Technology Directions Subcommittee for ISSCC from 2005 to 2008.

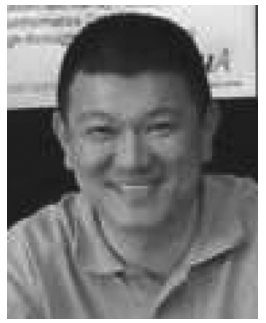

Patrick Yau received the B.Sc. and M.Sc. degrees from the University of Toronto, Toronto, ON, Canada, in 1987 and 1990, respectively, and the M.B.A. degree from York University, Toronto, in 2002.

$\mathrm{He}$ is currently the Manager of the University Health Network Microarray Centre, Toronto. His responsibilities include managing the production operation of microarrays, sales and marketing of contract genomics services, and technology development in functional genomics.

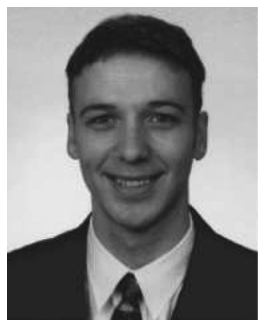

Roman Genov (S'96-M'02) received the B.S. degree (first rank) in electrical engineering from the Rochester Institute of Technology, Henrietta, NY, in 1996 and the M.S. and Ph.D. degrees in electrical and computer engineering from The Johns Hopkins University, Baltimore, MD, in 1998 and 2002, respectively.

He held engineering positions with Atmel Corporation, Columbia, MD, in 1995 and with Xerox Corporation, Rochester, NY, in 1996. He was a Visiting Researcher with the Laboratory of Intelligent Systems, Swiss Federal Institute of Technology (EPFL), Lausanne, Switzerland, in 1998 and with the Center for Biological and Computational Learning, Massachusetts Institute of Technology, Cambridge, in 1999. He is currently an Associate Professor with the Department of Electrical and Computer Engineering, University of Toronto, Toronto, ON, Canada. His research interests include analog and digital VLSI circuits, systems and algorithms for energy-efficient signal processing with applications to electrical, chemical and photonic sensory information acquisition, biosensor arrays, brain-silicon interfaces, parallel signal processing, adaptive computing for pattern recognition, and implantable and wearable biomedical electronics.

Dr. Genov was the recipient of the Canadian Institutes of Health Research Next Generation Award in 2005, the Brian L. Barge Award for excellence in microsystems integration in 2008, the DALSA Corporation Award for excellence in microsystems innovation in 2006 and 2009, and the Best Paper Award on sensors and Best Student Paper Award at the IEEE International Symposium on Circuits and Systems in 2009. He is an Associate Editor of the IEEE TRANSACTIONS ON BIOMEDICAL Circuits AND SYSTEMS, the IEEE TRANSACTIONS ON CIRCUITS AND SYSTEMS-II: EXPRESS BRIEFS, and IEEE SigNAL PROCESSING LETTERS. 